\title{
IS THE AKR CYCLOTRON MASER INSTABILITY A SELF-ORGANIZED CRITICALITY SYSTEM?
}

\author{
M. Marek*, and R. Schreiber*
}

\begin{abstract}
Data gathered by the POLRAD swept frequency radiospectrograph (Interball-2 mission) have been used for a preliminary analysis of a number of short bursts of the Auroral Kilometric Radiation (AKR) as a function of their intensity. The AKR intensity samples consisted of data snapshots integrated over $6 \mathrm{~ms}$ time periods. The histograms based on 53 data sets, containing up to several thousand samples, exhibit a power-law fall for higher intensities, characteristic for Self--Organized Criticality (SOC). The scaling parameter $\alpha$ varies for most cases between 2 and 3 with the dominant value 2.5 , and an error of the order of 0.1 . The SOC approach has already been used for an interpretation of some magnetospheric processes, but never for AKR.
\end{abstract}

\section{Interball-2 POLRAD experiment}

The Interball-2/Auroral Probe mission was launched on 29 August 1996 with an apogee of $19,140 \mathrm{~km}$, a perigee of $772 \mathrm{~km}$, and an orbital inclination of $62.8^{\circ}$. One of the onboard instruments (POLRAD swept-frequency spectro-polarimeter) was designed for an observation of the Auroral Kilometric Radiation (AKR). Linear sweeps usually covered the $4 \mathrm{kHz}-1 \mathrm{MHz}$ frequency range. The typical sweep duration was $6 \mathrm{~s}$, and the frequency resolution was $4 \mathrm{kHz}$ [Hanasz et al., 1998]. The $4 \mathrm{kHz}$ wide filter remained on the same frequency for $25 \mathrm{~ms}$, but the signal was integrated during the last $6 \mathrm{~ms}$.

The receiver had a $63 \mathrm{~dB}$ power spectral density (PSD) dynamic range. The sensitivity was about $2.5 \cdot 10^{-20} \mathrm{~W} /\left(\mathrm{m}^{-2} \mathrm{~Hz}\right)$. The AKR dynamic range expected for the POLRAD experiment could attain more than $80 \mathrm{~dB}-$ up to about $10^{-12} \mathrm{~W} /\left(\mathrm{m}^{-2} \mathrm{~Hz}\right)$. In order to suppress possible nonlinear effects in the receiver, the signal was attenuated by programmable attenuators $(20 \mathrm{~dB}, 40 \mathrm{~dB}$, or $60 \mathrm{~dB})$. We looked for a strong AKR, and for all 53 cases analyzed in this paper, a $20 \mathrm{~dB}$ attenuator was switched on. Therefore, the corresponding PSD dynamical range extended from about $10^{-18} \mathrm{~W} /\left(\mathrm{m}^{-2} \mathrm{~Hz}\right)$ to

${ }^{*}$ Space Research Centre, Polish Academy of Sciences 
$10^{-12} \mathrm{~W} /\left(\mathrm{m}^{-2} \mathrm{~Hz}\right)$. The antenna system consisted of two crossed electric dipoles $(22 \mathrm{~m}$ tip-to-tip) and one monopole $(11 \mathrm{~m})$. POLRAD was working only when the spacecraft was located in the northern hemisphere above the radiation belts (at invariant latitudes between $60^{\circ}$ and $82^{\circ}$ and at altitudes from $11,000 \mathrm{~km}$ to $19,000 \mathrm{~km}$ ). POLRAD was operational until 30 May 1998.

\section{Histograms of the electric power spectral density of short AKR bursts}

The AKR dynamic spectra of the PSD registered by the POLRAD experiment consisted of the spectral bins $4 \mathrm{kHz} \times 6 \mathrm{~ms}$. We will call them short AKR bursts. The PSD for adjacent bins was varying, sometimes up to $20 \mathrm{~dB}$.
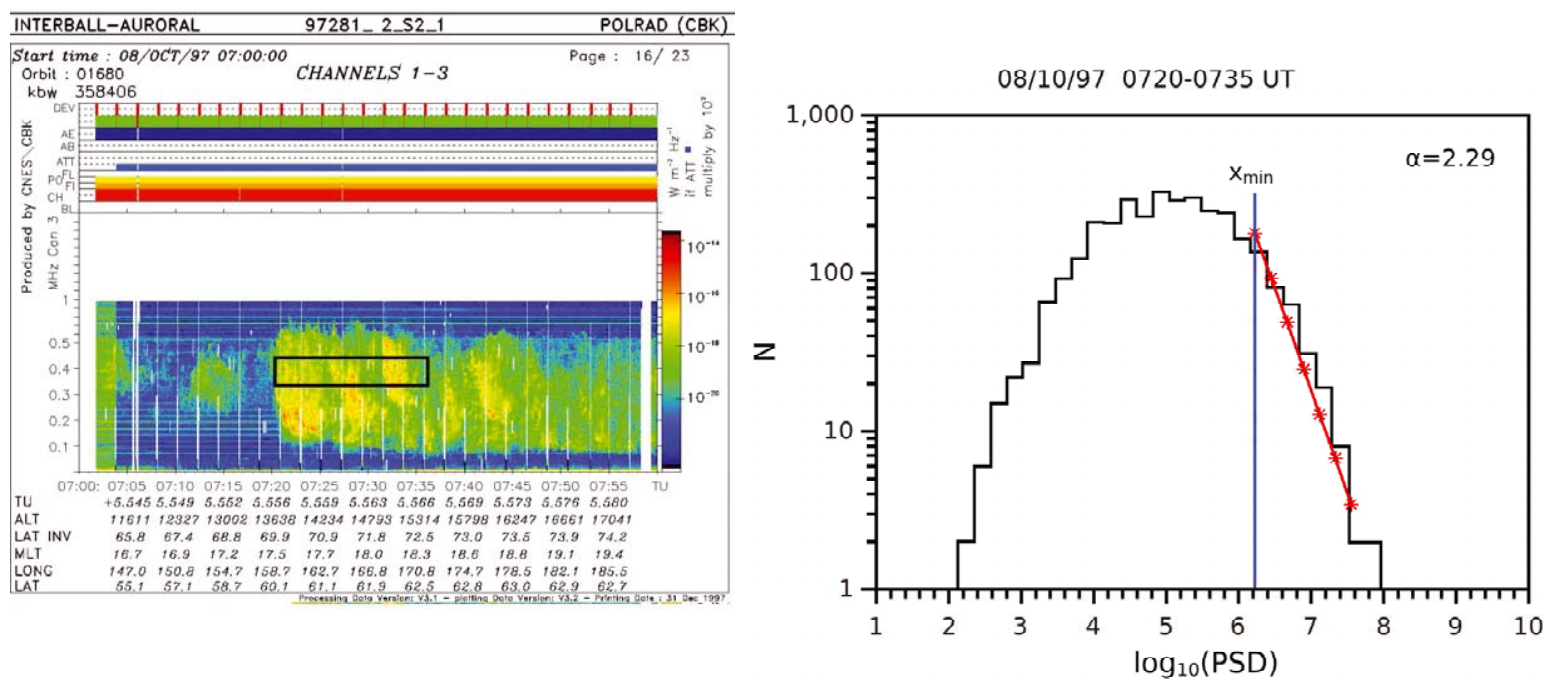

Figure 1: (Left) Example of an AKR dynamic spectrum. The data used for histogram generation are located inside the black frame $(15 \mathrm{~min} \times 100 \mathrm{kHz})$. The color bar labels should be multiplied by $10^{2}$ because a $20 \mathrm{~dB}$ attenuator was switched on. (Right) The corresponding histogram (in a $\log -\log$ scale) of 3240 measurements of the relative PSD of a short AKR bursts with a superposed red line produced by a least-squares fit to the distribution's tail. A vertical blue $x_{\text {min }}$ line delimits the right part of the histogram obeying a power-law behavior.

Figure 1 shows a quick look plot of a typical AKR dynamic spectrum, and the histogram in a $\log -\log$ scale of the number $N$ of AKR bursts as a function of the relative PSD (with respect to the $10^{-20} \mathrm{~W} /\left(\mathrm{m}^{-2} \mathrm{~Hz}\right)$ level). We found that above some PSD level $x_{\min }$, tails of the histograms in $\log -\log$ scale can be fitted by a straight line - they exhibit a power-law of the form:

$$
N(P) \propto P^{-\alpha}
$$

with $N(P)$ - count for histogram bin centered on the PSD equal $P, \alpha$ - exponent or scaling 
parameter. The histogram bins are spaced logarithmically - that means less fluctuations in the tail of the histogram.

We have chosen $100 \mathrm{kHz} \times 15$ minutes data blocks for three reasons: (a) in order to have a sufficient number $\mathrm{M}$ (up to a few hundred) measurements for $P S D>x_{\text {min }}$, (b) to have a relatively small displacement of the s/c on its orbit, and (c) to sample the AKR source region with a maximum vertical extent of $1000 \mathrm{~km}$. The last number results from constraints imposed by conditions (a) and (b). We checked $\alpha$ values for different frequency ranges (between 100 and $500 \mathrm{kHz}$ ) - no $\alpha$ dependency on the frequency was found.

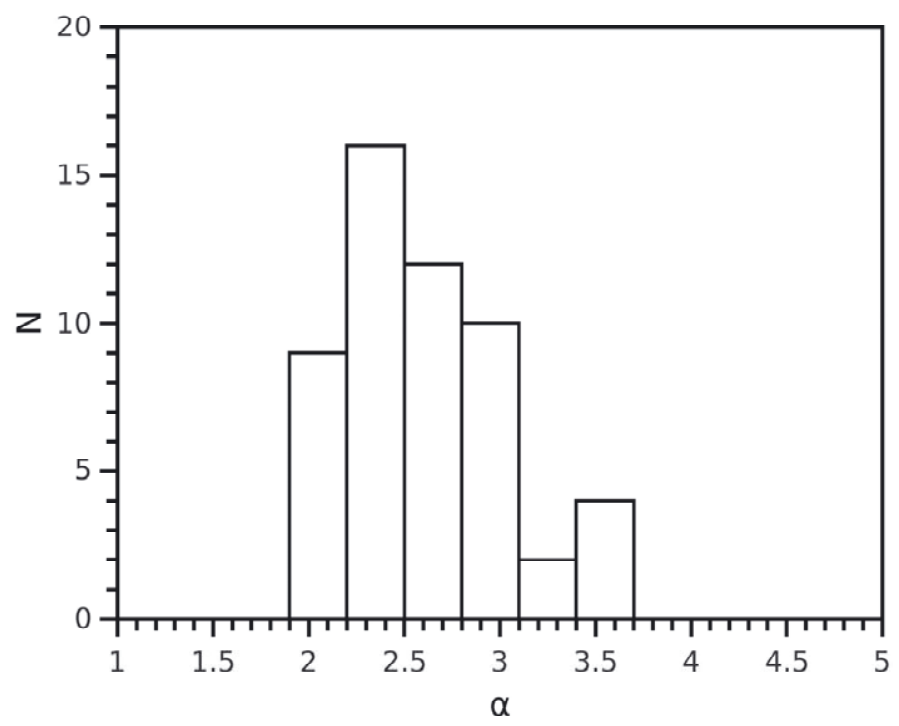

Figure 2: Histogram of $\alpha$ values estimated for 53 histograms of the short AKR bursts PSD.

Figure 2 shows a histogram of the power-law scaling parameter $\alpha$ estimated from 53 AKR dynamic spectra (and subsequently calculated histograms). The histograms (like in Figure 1) are typically produced for about 3000 PSD measurements. On the other hand, there are not many bins in the power-law part of the histograms, and the last bins containing rare events are prone to significant fluctuations. Determining the location of the part of the histogram displaying a power-law behavior is therefore very subjective.

\section{Getting $\alpha$ from the data}

\subsection{Clauset et al. [2009] approach}

A more objective and precise method for a determination of the power-law scaling parameter $\alpha$ has been employed with solar data by D'Huys et al. [2016].

A detailed presentation and justification of the new method can be found in a review paper published by Clauset et al. [2009]. They propose the maximum likelihood estimator 
(MLE) for the data drawn from the distribution obeying a power-law. For our short AKR bursts the power-law distribution starts from some $x_{\min }$ value (for $x \geq x_{\min }$ ) and contains $M$ data samples:

$$
\alpha=1+M\left[\sum_{i=1}^{M} \ln \frac{x_{i}}{x_{m i n}}\right]^{-1}
$$

The $\alpha$ symbols correspond here to the estimates calculated from the data. The standard error $\sigma$ of $\alpha$ has the following form:

$$
\sigma=\frac{\alpha-1}{\sqrt{M}}+O(1 / M)
$$

Looking at the histograms, we can try to delimit parts exhibiting a power-law fall with growing $x$. It can be very subjective, especially for histograms with a small number of bins. Clauset et al. [2009] propose a determination of $x_{\text {min }}$ by employing a goodness-of-fit test based on the Kolmogorov-Smirnov statistic/metrics. Other metrics, like AndersonDarling or Kuiper [Press et al., 2007] can also be applied. In this new approach we determine the scaling parameter $\alpha$ using every data sample $x_{i}$ - they are not binned as in the histogram.

\subsection{Some statistical results obtained with a new approach}

A detailed analysis of heavy-tailed distributions can be quite complicated. Recently Alstott et al. [2014] released into the public domain a flexible implementation of the Clauset et al. [2009] ideas - a Python package named "powerlaw". We used it for most calculations in the present paper.

We compared $\alpha$ and $\sigma$ values calculated for $x_{\min }$ values determined for three different metrics: Kolmogorov-Smirnov, Anderson-Darling and Kuiper. Results produced by Kolmogorov-Smirnov and Kuiper metrics are similar, but for Anderson-Darling metric, the histogram of $\alpha$ values is much more compact (Figure 3). A similar rule applies to the scatter plot of $\sigma$ vs. $\alpha$ (Figure 4 ).

For each of the 53 analyzed AKR dynamic spectra we have a different number $M$ of intensity samples fitted to the power-law distribution. A comparison of the $\sigma$ vs. $M$ dependency in Figure 5 shows once more that the Anderson-Darling metric is a better choice than the Kolmogorov-Smirnov or Kuiper metrics. We have more data points for the determination of $\alpha$ and $\sigma$, and the errors are smaller. Clauset et al. [2009] suggest that $M \geq 50$ is a reasonable rule of thumb for extracting reliable parameter estimates. That rule is also valid for our data, but for the Anderson-Darling metric the smallest values of $M$ are even greater than 100 .

We compared the power-law distribution hypothesis with two alternative hypotheses: the exponential and $\log$-normal distributions, calculating the significance value $p$ and the loglikelihood ratio $R$ between two distributions as proposed by Clauset et al. [2009]. Direct definitions taken from the "power-law" code are as follows: 

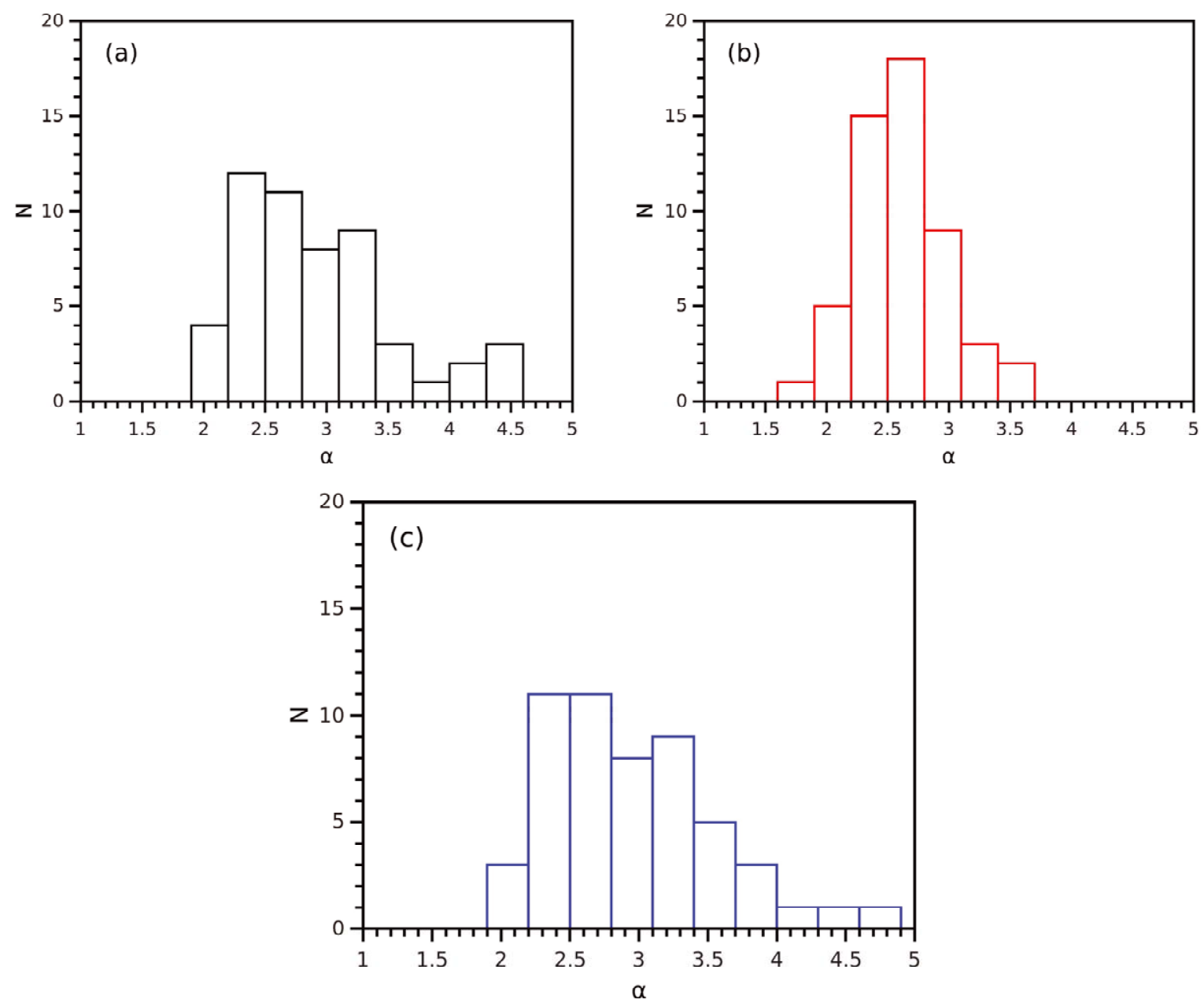

Figure 3: Histograms of MLE estimates of the $\alpha$ scaling parameter based on $x_{\text {min }}$ values calculated for three different metrics: (a) Kolmogorov-Smirnov, (b) Anderson-Darling, and (c) Kuiper.

- $R$, the loglikelihood ratio of the two sets of likelihoods. If positive, the first set of likelihoods is more likely (and so the probability distribution that produced them is a better fit to the data). If negative, the reverse is true.

- $p$, the significance of the sign of $R$. If below a critical value (typically .05), the sign of $R$ is taken to be significant. If above the critical value, the sign of $\mathrm{R}$ is taken to be due to statistical fluctuations.

Figure 6 presents the results of such comparisons for our data $\left(x_{\min }\right.$ calculated for AndersonDarling metric).

The power-law distribution is a better fit to our data than the exponential distribution, but for the power-law/log-normal pair there is no clear conclusion with our not so numerous and rather noisy data samples. Our receiver integrated the signal in the $4 \mathrm{kHz}$ band during $6 \mathrm{~ms}$. For such a configuration we have PSD fluctuations of the order of 

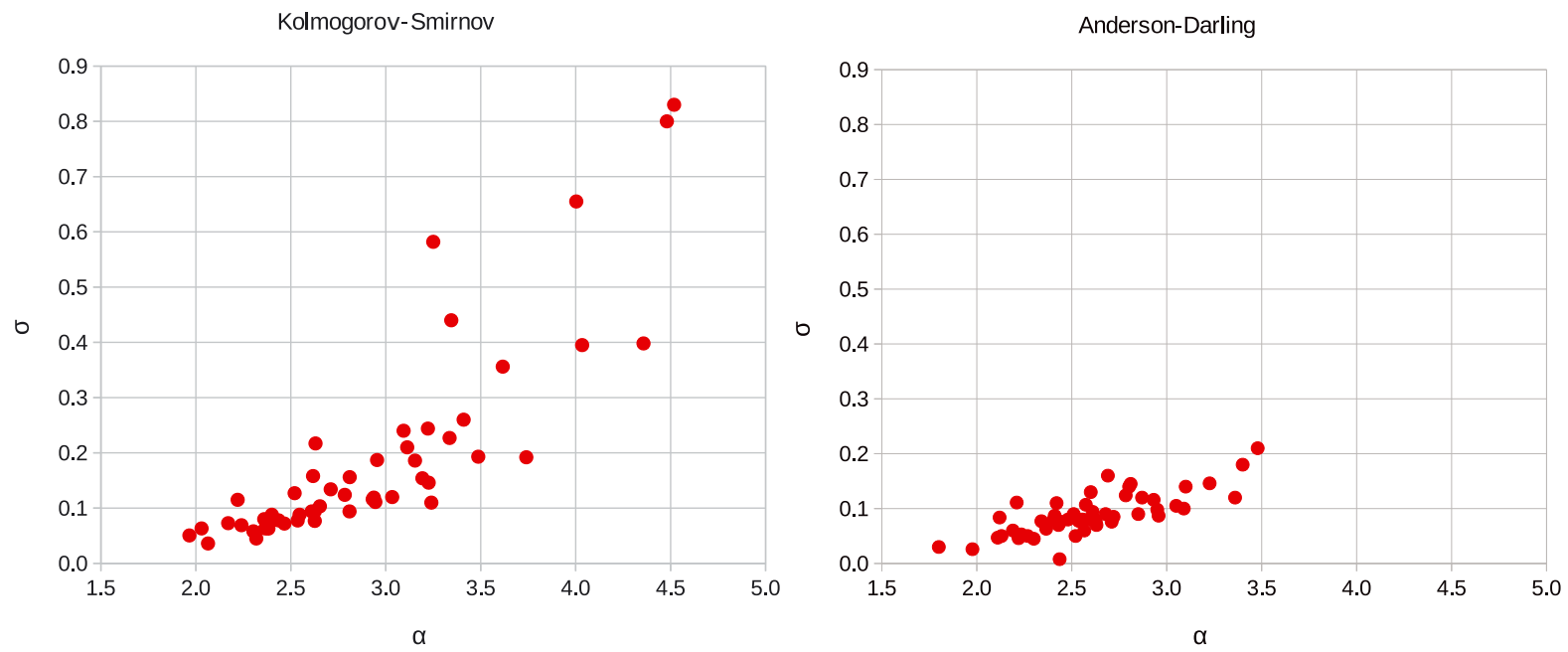

Figure 4: Scatter plot of $\sigma$ vs. $\alpha$ values calculated using the MLE estimator. (Left) For Kolmogorov-Smirnov metric and (Right) for Anderson-Darling metric.
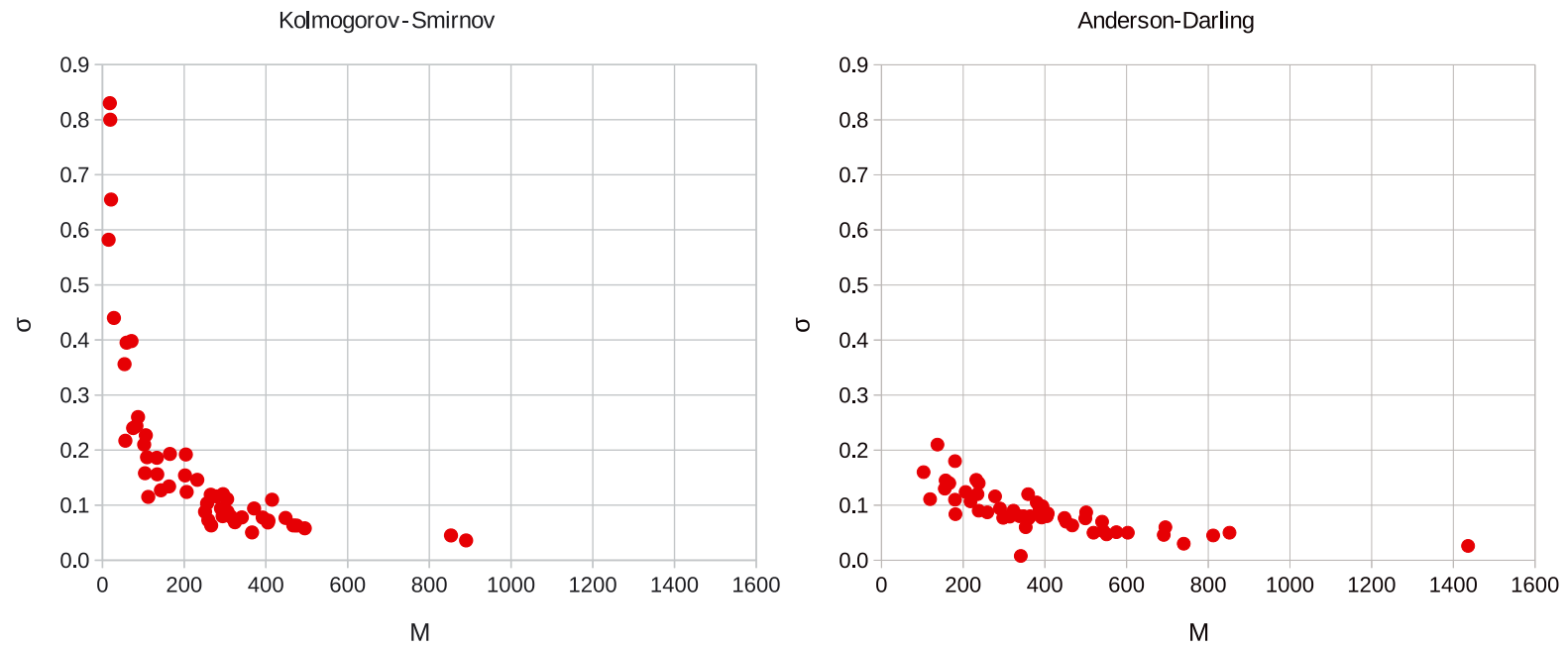

Figure 5: Scatter plot of $\sigma$ vs. the number of AKR data samples $(M)$ that are more intense than $x_{\text {min }}$ (calculated with MLE estimator). (Left) For Kolmogorov-Smirnov metric and (Right) for Anderson-Darling metric.

$20 \%$. The signal at the output of our receiver was proportional to the logarithm of the input PSD and the usable telemetry range covered a $63 \mathrm{~dB}$ dynamic range of the receiver, which corresponds to about 100 telemetry units. $0.63 \mathrm{~dB}$ corresponds to the PSD ratio between successive units of about 1.16. A similar inconclusive situation has been recently reported by Riley and Love [2017] for the Dst index.

The scaling factor $\alpha$ has a dominant value of $\sim 2.5$ with a standard error of $\sim 0.1$, and about $80 \%$ of all $\alpha$ values can be found in the interval $[2.0,3.0]$. 

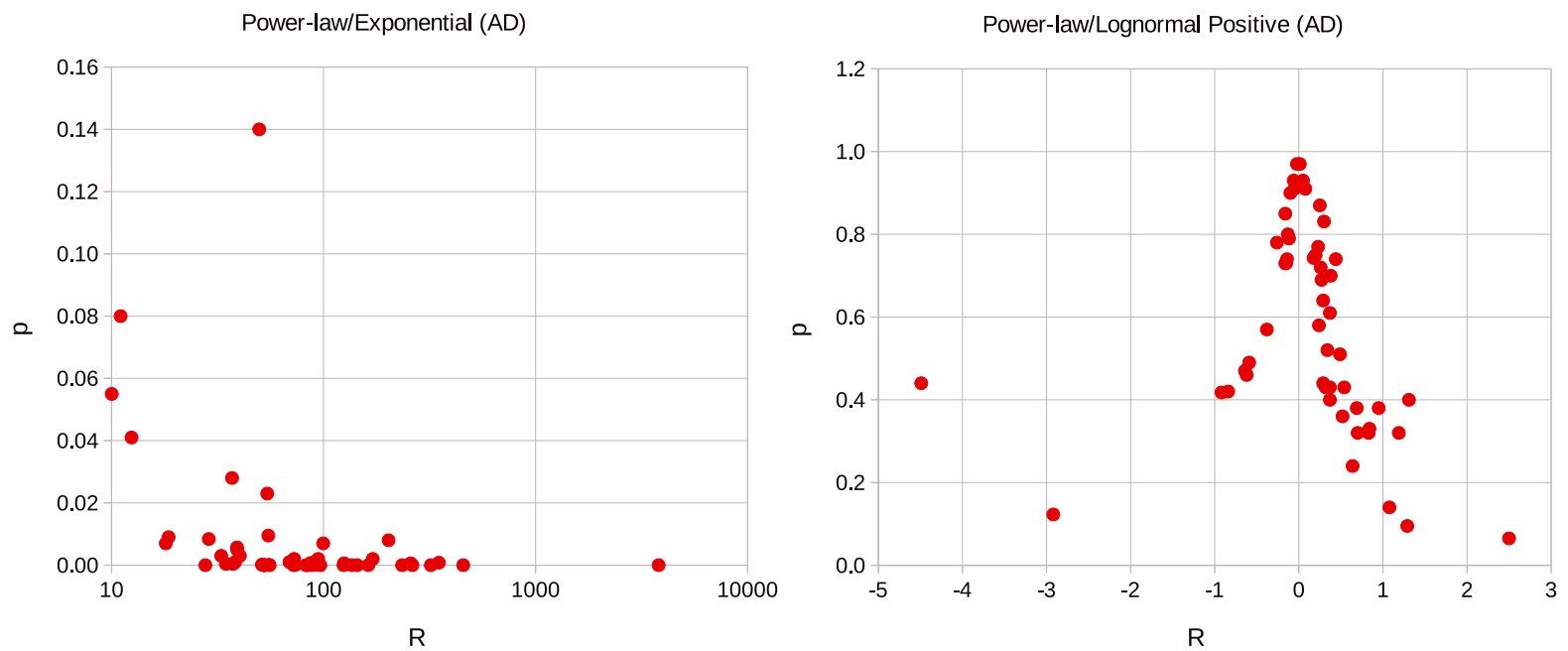

Figure 6: The significance value $p$ vs. the loglikelihood ratio $R$ between two distributions: power-law and exponential (Left), power-law and log-normal positive (Right).

\section{$4 \quad$ AKR and SOC?}

The power-law distribution observed in AKR dynamic spectra points toward an applicability of the Self-Organized Criticality (SOC) concept for an analysis of the AKR generation process. A general physics-based definition of SOC was given by Aschwanden [2014]:

$S O C$ is a critical state of a nonlinear energy dissipation system that is slowly and continuously driven towards a critical value of a system-wide instability threshold, producing scale-free, fractal-diffusive, and intermittent avalanches with power-law-like size distributions.

SOC behavior is observed for many physical systems [Bak, 1996], also in Solar Physics and Astrophysics [Aschwanden et al., 2016]. The SOC concept can be applied to the Cyclotron Maser Instability (CMI) responsible for the AKR generation as well. We observe the AKR radiating region consisting of a bunch of small elementary AKR sources which are located within the auroral oval [Mutel et al., 2008; Schreiber et al., 2017]. After reaching the threshold of CMI instability in the source region, we observe many elementary AKR sources "firing" with different intensities - that corresponds to the avalanche in the SOC definition. The increment of the CMI instability can depend on the stability of the electron distribution function as well as on the convective growth length [Mutel et al., 2007].

\section{Discussion}

The power-law distributions of the PSD of short AKR bursts should be confirmed for a greater amount of data, but the estimates of the scale parameter $\alpha$ based on our relatively small pool of data are also interesting. Our scaling factors $\alpha$ have a dominant value of 
$\sim 2.5$ and, about $80 \%$ of all $\alpha$ can be found in the interval $[2.0,3.0]$. On the other hand, similar values of $\alpha$ were found, for example, for solar microwave bursts (1.2-2.5) - Table 15 in Aschwanden et al. [2016]. Another example - solar decimetric millisecond spikes with $\alpha=2.99 \pm 0.63$ (most probably produced by the CMI instability, like AKR), was published by Aschwanden et al. [1998]. The next step consists in finding the bridge between generalized mathematical models and the physics behind our statistics. This is not an easy task. We have to look at the existing models and ways of SOC simulation. We shall start with a simple Logistic Growth Model parametrizing the shape and amplitude of the burst (rise, saturation, and decay) [Aschwanden, 2011] and generating corresponding statistics. We are also interested in possible seasonal changes of $\alpha$. We made such checks, but for $\alpha$ determined with low precision from the histograms no seasonal dependency was found.

We also hope that a greater amount of data and complementary statistical analysis using alternative approaches (like Bayesian methods) will help to clarify the inconclusive comparison of fits for power-law and log-normal distributions.

\section{Conclusions}

We investigated 53 cases of AKR dynamic spectra gathered by the Interball-2 POLRAD experiment and found a power-law distribution for intensities of strong, short AKR bursts. We applied to our data quite recent statistical methods superior to the typical approach based on data histogram analysis. These methods give reliable results even for not very numerous data samples and avoid information loss caused by binning of the data. The scaling factors $\alpha$ have a dominant value of $\sim 2.5$ with a standard error of $\sim 0.1$, and about $80 \%$ of all $\alpha$ can be found in the interval [2.0,3.0]. A next step will consist in adjusting results for simple, analytic SOC models to the AKR source sizes, AKR burst parameters, and such Cyclotron Maser parameters as CMI growth rate or power gain. We also want to improve our statistics using more data and to check alternative approaches to the statistical analysis of our data.

Acknowledgments. This research was supported by the Ministry of Science and Higher Education and the National Science Center in Poland under grant 2012/07/B/ST9/04414 (RS), and the Ministry of Science and Higher Education via financing of development of young researchers and doctoral program participants (MM). The Editors thank Spencer Hatch and one anonymous reviewer for their help in evaluating this paper.

\section{References}

Alstott, J., E. Bullmore, and D. Plenz, Powerlaw: A Python package for analysis of heavytailed distributions, PLoS ONE, 9(1), e85777, doi:10.1371/journal.pone.0085777, 2014 .

Aschwanden, M. J., B. R. Dennis, and A. O. Benz, Logistic avalanche processes, elementary time structures, and frequency distributions of flares, Astrophys. J, 497, 2, 
972-993, 1998.

Aschwanden, M. J., Self--Organized Criticality in Astrophysics, Springer-Verlag, Berlin, Heidelberg, Germany, 2011.

Aschwanden, M. J., A macroscopic description of self-organized systems and astrophysical applications. Astrophys. J., 782, id.54, doi:10.1088/0004-637X/782/1/54, 2014.

Aschwanden, M. J., N.B. Crosby, M. Dimitropoulou, M. K. Georgoulis, S. Hergarten, J. McAteer, A. V. Milovanov, S. Mineshige, L. Morales, N. Nishizuka, G. Pruessner, R. Sanchez, A. S. Sharma, A. Strugarek, and V. Uritsky, 25 years of self-organized criticality: Solar and astrophysics. Space Sci. Rev., 198, 47-166, doi:10.1007/s11207016-0910-5, 2016.

Bak, P., How nature works: The science of self-organized criticality, Springer-Verlag, New York, USA, 1996.

Clauset, A., C.R. Shalizi, and M.E. J. Newman, Power-law distributions in empirical data, SIAM Rev., 51, 661-703, doi:10.1137/070710111, 2009.

D'Huys, E., D. Berghmans, D. B. Seaton, and S. Poedts, The effect of limited sample sizes on the accuracy of the estimated scaling parameter for power-law-distributed solar data, Solar Phys., 291, 1561-1576, doi:10.1007/s11207-016-0910-5, 2016.

Hanasz, J., Z. Krawczyk, M. M. Mogilevsky, R. Schreiber, H. de Feraudy, K. Dudzinski, T. V. Romantsova, W. Nowakiewicz, A. Kraynyuk, M. Barylko, A. Buczkowska, J. Juchniewicz, V. N. Nazarov, and N. Mikhalev, Observation of auroral kilometric radiation on the INTERBALL-2 satellite: the POLRAD experiment, Cosmic Res., 36, 617, 1998.

Mutel, R. L., W. M. Peterson, T. R. Jaeger, and J. D. Scudder, Dependence of cyclotron maser instability growth rates on electron velocity distributions and perturbation by solitary waves, J. Geophys. Res., 112, A07211, doi:10.1029/2007JA012442, 2007.

Mutel, R. L., I. W. Christopher, and J. S. Pickett, Cluster multispacecraft determination of AKR angular beaming, Geophys. Res. Lett., 35, L07104, doi:10.1029/2008GL033377, 2008.

Press, W. H., S. A. Teukolsky, W. T. Vetterling, and B. P. Flannery, Numerical Recipes: The Art of Scientific Computing (3rd Ed.), Cambridge University Press, New York, USA, 2007.

Riley, P., and J. J. Love, Extreme geomagnetic storms: Probabilistic forecasts and their uncertainties, Space Weather, 15, 53-64, doi:10.1002/2016SW001470, 2017.

Schreiber, R., M. Panchenko, J. Hanasz, R. Mutel, and I. Christopher, Beaming of intense AKR seen from the Interball-2 spacecraft, J. Geophys. Res., 122, 249-257, doi:10.1002/2015JA022197, 2017. 
